\title{
Preferencias y rendimiento de los alumnos en el aprendizaje de Investigación Operativa con videos
}

\author{
Marina Segura, Concepción Maroto, Concepción Ginestar, José Ramón Navarro \\ Departamento de Estadística e Investigación Operativa Aplicadas y Calidad. Universitat \\ Politècnica de València. E-mail: masema@upvnet.upv.es; cmaroto@eio.upv.es; \\ cginesta@upvnet.upv.es; jonacer@upvnet.upv.es
}

\begin{abstract}
The increasing supply of videos as an online resource has changed the performance of students who study degrees based on face-to-face learning. The objectives of this paper are to elicit current preferences for different types of learning resources, especially videos, developed to teach Operations Research in Business Administration and Management degree, as well as analysing the relation between the videos use and students' performance. The methodology is based on a survey using the Likert scale and the multicriteria method AHP.

While all available resources are used to learn the subject, the results show a predominant preference for exam answers and videos, although more than one third of the students also use books and slides. Detailed exam answers improve learning and decrease tutorial appointments. There are significant differences in the use of learning resources among groups and resources affect students' behaviour and performance. The videos with teaching modules decrease face-to-face class attendance, while the videos with interactive case studies affect performance positively. Therefore, we think that moving forward in this line would improve effective learning.
\end{abstract}

Keywords: learning, videos, students' preferences, teaching resources, active learning, autonomous learning

\section{Resumen}

La creciente oferta de videos como recurso online ha modificado el comportamiento de los alumnos que cursan titulaciones con docencia presencial. Los objetivos de este trabajo son cuantificar las preferencias actuales por los diversos tipos de materiales, y en particular los videos, elaborados para la docencia de Investigación Operativa en el Grado en Administración y Dirección de Empresas, así como analizar la relación entre el uso de videos y el rendimiento de los alumnos. La metodología se basa en una encuesta con la escala de Likert y la técnica AHP.
} 
Si bien todos los recursos disponibles se utilizan para preparar la materia, los resultados muestran una preferencia mayoritaria por los exámenes resueltos y videos, aunque más de un tercio de los alumnos también utilizan libros y presentaciones. Los exámenes resueltos mejoran el aprendizaje y reducen las tutorías. Se han encontrado diferencias significativas en el uso de los recursos por grupo y los medios utilizados también afectan al comportamiento y al rendimiento de los alumnos. Los videos con unidades didácticas reducen la asistencia a clase presencial, mientras que los videos de casos prácticos interactivos afectan positivamente al rendimiento. Por tanto, creemos que avanzar en esta última línea mejoraría el aprendizaje efectivo.

Palabras clave: aprendizaje, videos, preferencias, recursos, enseñanza, aprendizaje activo, aprendizaje autónomo.

\section{Introducción}

La creciente oferta de formación online y semipresencial en las universidades ha generado una gran cantidad y diversidad de recursos docentes. Esta tendencia también se ha constatado en los estudios de gestión y administración de empresas (Arbaugh et al., 2009). La Universitat Politècnica de València (UPV) tiene una larga trayectoria en este sentido y en particular en el desarrollo de videos como recursos de aprendizaje, que son cada vez más demandados por los alumnos de docencia presencial.

En estudios previos hemos constatado que los alumnos de docencia inversa son los que mayor preferencia muestran por los videos para aprender Investigación Operativa y los que más valoran este recurso, que está influyendo en el comportamiento de los alumnos de docencia presencial. Así, los videos inicialmente grabados para apoyar la docencia inversa amplían las oportunidades de aprendizaje y contribuyen a adquirir la competencia de aprendizaje autónomo también del alumno presencial (Bas et al., 2017). Por una parte, la disponibilidad de videos reduce la necesidad de tutorías al poder visualizarlos varias veces, tomar notas y en definitiva adaptarlos a las necesidades personales. Por otra, los videos pueden reducir la asistencia a clase tal y como se ha observado también en otros trabajos, que analizan el impacto de los videos como material complementario a las clases presenciales (Winch y Cahn, 2015; Sharkey y Nurre, 2016). Además, en Bas et al. (2017) se encontró una relación negativa entre utilización de videos y rendimiento en la asignatura, que se consideró debido a la carencia de videos con casos prácticos, que sí se trabajan en el aula y no se resolvían de forma autónoma por los alumnos que utilizan más los videos. Para comprender los conceptos de Investigación Operativa es esencial que el alumno los aplique a casos prácticos, bien en clase o de forma autónoma.

Mejorar estos resultados y las sugerencias de los alumnos nos animaron a solicitar un Proyecto de Innovación y Mejora Educativa en la UPV (PIME B01) para el curso 2017-18, titulado "Casos y aplicaciones de toma de decisiones: vídeos y obtención de preferencias de los alumnos mediante técnicas multicriterio", cuyos primeros resultados presentamos en este trabajo.

(cc) EY-NC-ND 2018, Universitat Politècnica de València

Congreso IN-RED (2018) 


\section{Objetivos}

Teniendo en cuenta la experiencia previa comentada en la introducción los objetivos del presente trabajo son los siguientes:

1. Cuantificar las preferencias de los alumnos por los distintos tipos de materiales elaborados para la docencia de Investigación Operativa en el Grado en Administración y Dirección de Empresas y en particular los videos.

2. Analizar la relación entre el grado de utilización de videos y el rendimiento de los alumnos.

Para alcanzar el primer objetivo se ha realizado y analizado una encuesta a los alumnos que incluye tanto la escala de Likert, metodología habitual en este tipo de estudios, como la técnica multicriterio Analytic Hierarchy Process (AHP), muy empleada para elicitar las preferencias en toma de decisiones colaborativa y que representa un enfoque novedoso en educación (Saaty y Vargas, 2001; Saaty y Peniwati, 2008). Para alcanzar el segundo objetivo se han utilizado test estadísticos adecuados como Chi-cuadrado de Pearson en las tablas de contingencia, test de Kruskal-Wallis y test de Mann-Whitney para el análisis de variables cuantitativas.

En los siguientes apartados se presenta el contexto y el material docente previo al desarrollo de nuevos videos con casos prácticos y aplicaciones reales, la metodología y los principales resultados de la encuesta realizada a los alumnos la última semana del curso (adjunta como anexo). Por último, se incluyen las conclusiones del estudio y futuras líneas de mejora educativa.

\section{Desarrollo de la innovación}

\subsection{Contexto}

La Investigación Operativa es una materia de naturaleza cuantitativa que se imparte en $3^{\circ}$ curso del Grado en ADE (Facultad de Administración y Dirección de Empresas, UPV). En síntesis, los resultados de aprendizaje son que los alumnos sean capaces de formular modelos de optimización para resolver problemas reales de toma de decisiones, aplicar los conceptos y algoritmos para resolver modelos de programación lineal, entera, no lineal, multiobjetivo y multicriterio mediante software profesional e interpretar los resultados para mejorar las decisiones empresariales.

La asignatura tiene 3 créditos de teoría y 3 créditos de prácticas, 1 de prácticas de aula y 2 de prácticas informáticas. Se utiliza una metodología orientada hacia el aprendizaje, que tiene su origen en varios Planes de Innovación Educativa desarrollados en la UPV (Maroto et al., 1997; Alcaraz et al., 2003; Alcaraz et al., 2004).

El sistema de evaluación se basa en la evaluación continua de las prácticas informáticas (20\%) y 2 exámenes (40\% cada uno). Los alumnos que no superan la asignatura pueden realizar un examen de recuperación. La media de la tasa de rendimiento de los estudiantes 
en los 6 últimos cursos es 90\%. El porcentaje medio de suspensos es 7,8\% y el de no presentados $2,2 \%$.

\subsection{Material docente}

El material docente básico consiste en un libro de texto diseñado para impartir la asignatura en el grado en ADE, las presentaciones del profesor en Powerpoint, videos con la voz del profesor explicando las presentaciones utilizadas en clase presencial y todos los exámenes resueltos (6 cursos). Tanto el libro como las presentaciones y los exámenes están en castellano y en inglés, ya que un grupo -Alto Rendimiento Académico (ARA)- se imparte en inglés. El libro está disponible en formato papel y electrónico (Maroto et al., 2012, Maroto et al., 2014). Los alumnos tienen acceso a 17 vídeos en castellano de toda la asignatura con una duración entre 8 min y 55 (grabados en 2015 para un proyecto piloto de docencia inversa) y a 3 en inglés (https://media.upv.es).

Dado que el material incluye casos prácticos a desarrollar y resolver en el aula y en el laboratorio informático, los alumnos no disponen de la solución, siendo ésta una demanda constatada en estudios previos (Bas et al., 2017), que se ha tratado de cubrir con nuevos videos en el curso 2017-18.

Durante el curso 2017-18 se han elaborado 12 nuevos videos con casos prácticos y aplicaciones reales (https://media.upv.es). Estos videos cumplen los requerimientos que exige el programa Docencia en Red de la UPV. Concretamente, son interactivos, indivisibles e independientes y duran 10 minutos. Aunque se han diseñado para cubrir las necesidades de la asignatura Investigación Operativa en ADE, no están contextualizados y pueden utilizarse en otras titulaciones y universidades.

\subsection{Metodología}

Para conocer las preferencias de los alumnos por los videos comparados con otros recursos docentes y su utilidad para mejorar su aprendizaje y rendimiento se ha diseñado la encuesta que figura en el anexo. La encuesta se ha realizado a todos los alumnos de Investigación Operativa del grado en ADE durante el curso académico 2017-2018, que se distribuyen en 4 grupos (Mañanas, Tardes, ARA y Doble Titulación ADE-Telecomunicaciones).

Se ha preguntado por el grado de utilización del material docente en el aprendizaje de Investigación Operativa utilizando la escala de Likert de 1 a 5. También aplicamos el método AHP basado en comparaciones por pares y la escala de 1 a 9 para conocer las preferencias de los estudiantes, que es una técnica multicriterio contrastada para conocer y agregar las preferencias de grupos, muy utilizada en toma de decisiones colaborativa y con mayor poder discriminante que la escala de Likert (Bas et al. 2017).

La encuesta, realizada la última semana de curso, también pregunta por calificación obtenida en la asignatura (nota primer parcial), si asiste regularmente a las clases y la meta personal en cuanto a la puntuación final para estudiar la posible influencia de los videos en el comportamiento y rendimiento de los alumnos. Por último, la encuesta incluye una pregunta abierta sobre sugerencias de mejora de los materiales docentes.

(cc) EY-NC-ND 2018, Universitat Politècnica de València

Congreso IN-RED (2018) 


\section{Resultados}

La tabla 1 recoge el número de alumnos matriculados en cada grupo y el porcentaje de los que han respondido la encuesta, que a nivel global es del $82,2 \%$. Se puede afirmar que tenemos una elevada representatividad tanto a nivel global como en todos los grupos.

Tabla 1. Número de alumnos matriculados y porcentaje de respuesta por grupos

\begin{tabular}{|l|c|c|c|}
\hline \multicolumn{1}{|c|}{ Grupo } & Matriculados & Respuesta & \% Respuesta \\
\hline Mañanas & 68 & 54 & 79,4 \\
\hline Doble ADE_Teleco & 16 & 14 & 87,5 \\
\hline ARA & 31 & 20 & 64,5 \\
\hline Tardes & 37 & 32 & 86,4 \\
\hline TOTAL & 152 & 125 & 82,2 \\
\hline
\end{tabular}

Arbaugh et al. (2009) y Cochran (2012) defienden la utilización y complementariedad de metodologías cualitativas y cuantitativas en estudios de educación. Por una parte, existe una gran controversia sobre la naturaleza cualitativa o cuantitativa de la escala de Likert, mientras que los resultados que se obtienen con AHP son cuantitativos. Autores como Garuti (2017) defienden el carácter cualitativo de la escala de Likert. Sin embargo, en la literatura también se encuentran resultados de la escala de Likert con análisis propios de variables cuantitativas.

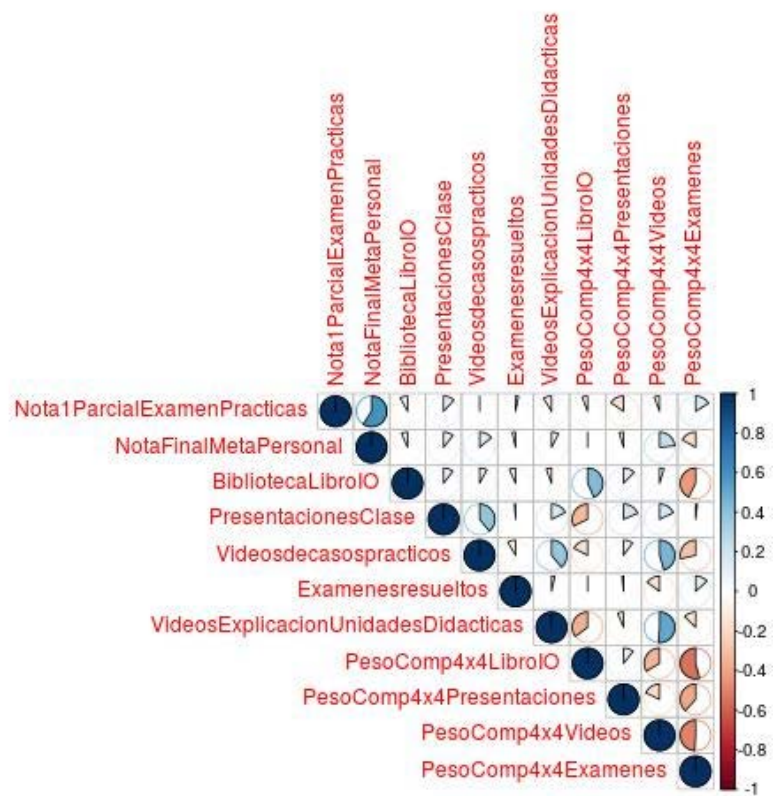

Fig. 1 Gráfico de correlaciones del rendimiento de los alumnos y grado de utilización de los recursos docentes

A nivel ilustrativo la figura 1 presenta un gráfico de correlaciones de las variables consideradas en la encuesta que muestra la elevada correlación entre la nota que han

(c) EY-NC-ND 2018, Universitat Politècnica de València

Congreso In-Red (2018) 
obtenido los estudiantes (primer parcial, 50\% de la calificación) y la meta personal que tienen para su calificación final, pudiendo ser éste un factor importante en la explicación del rendimiento, en especial para las notas más altas como sobresalientes y matrículas de honor. Además, destaca la inexistencia de correlación entre la utilización de los exámenes resueltos con ninguna otra variable.

Si evaluamos el grado de utilización de los diferentes tipos de materiales con el porcentaje de alumnos que los utiliza bastante o mucho, medido con la escala de Likert, el resultado de la encuesta refleja que el recurso más utilizado son los exámenes resueltos (92,00\%), seguido de los videos con explicación de las unidades didácticas $(54,17 \%)$ y videos de casos prácticos (50,41\%). El porcentaje de alumnos que utiliza bastante o mucho el libro de texto $(37,40 \%)$ y las presentaciones que utiliza el profesor en las clases $(37,19 \%)$ es menor, aunque importante porque representa más de un tercio del alumnado. En síntesis, se puede afirmar que todos los recursos disponibles se utilizan para preparar la asignatura.



Fig. 2 Utilización del material docente en el aprendizaje de Investigación Operativa en porcentaje de alumnos por grupo. Escala de Likert.

Las diferencias por grupo se pueden observar en la figura 2, que se han analizado mediante tablas de contingencia, como herramienta adecuada para variables cualitativas. Mediante el test Chi-Cuadrado de Pearson se han encontrado diferencias significativas en el caso del libro y las presentaciones al 94\% y $95 \%$ de confianza respectivamente. Los alumnos que cursan doble titulación ADE y Telecomunicación (DAT) destacan por ser los que en mayor proporción utilizan bastante o mucho el libro y a la vez no lo usa nunca en una proporción similar (42\%). Las diferencias en el uso de las presentaciones también se debe al distinto comportamiento de los alumnos de la doble titulación respecto a los demás, que son los que aprovechan más este recurso.

(c)) BY-NC-ND 2018, Universitat Politècnica de València

Congreso IN-RED (2018) 
Respecto a los exámenes y los videos, tanto de unidades didácticas como de casos prácticos las diferencias entre grupos no son significativas. Destaca que entre el 90 y 100\% de los alumnos utilizan bastante o mucho los exámenes resueltos. En cuanto a los videos la mitad de los estudiantes indican que los han utilizado bastante o mucho, siendo más empleados que el libro y las presentaciones.

Para obtener las preferencias de los alumnos por los diferentes materiales de Investigación Operativa en ADE utilizando la técnica multicriterio AHP en primer lugar se ha calculado la consistencia de las respuestas tanto de la matrices $4 \times 4$, como de las matrices $3 \times 3$, eliminando del análisis los exámenes. El porcentaje de encuestas consistentes del total de alumnos es 58\% de la matriz 3x3 y 62\% de la matriz 4x4, siendo mayor en DAT ( $79 \%$ en ambos casos), y menor en el grupo más numeroso de la mañana (52\% en ambos casos).

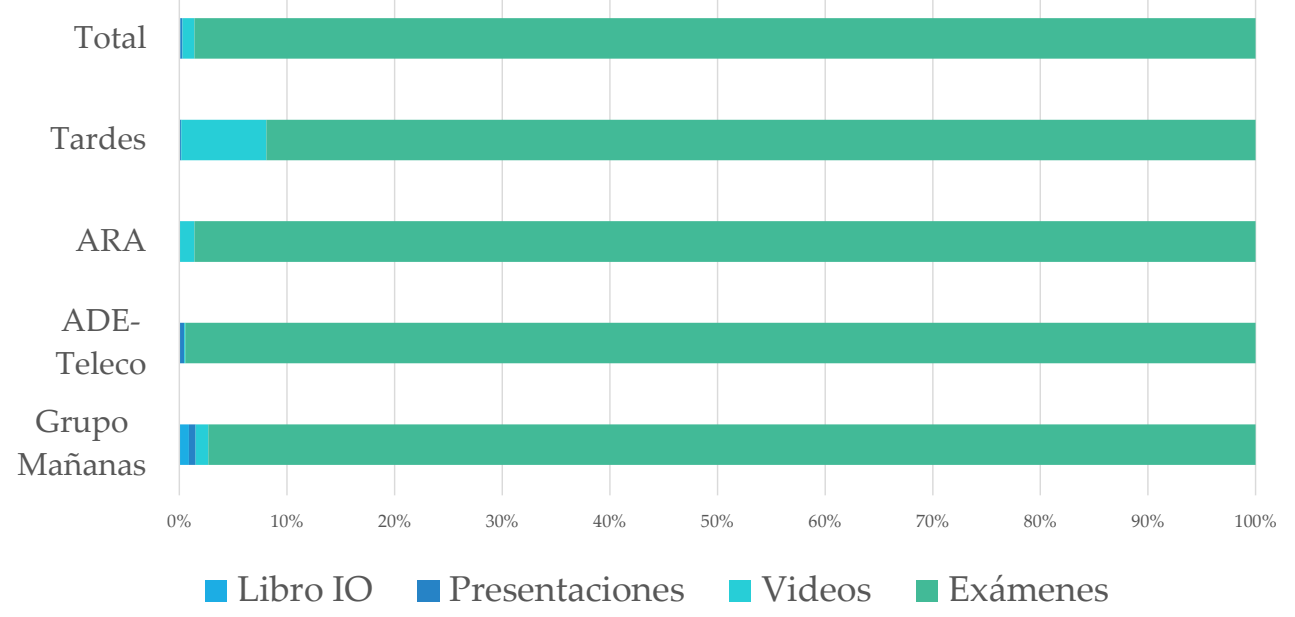

Fig. 3 Preferencias agregadas de los alumnos por el material docente en el aprendizaje de Investigación Operativa obtenidas con AHP. El porcentaje indica el peso relativo de cada uno de los 4 recursos.

Utilizando sólo las respuestas con consistencia aceptable (índice de inconsistencia menor o igual a 0,10 ) se han obtenido los resultados que se presentan en la figuras 3 y 4 . Los pesos de cada grupo para los recursos se han calculado agregando las comparaciones por pares realizadas por los alumnos mediante la media geométrica y de esta manera determinar la preferencia del grupo. La media geométrica mantiene la consistencia de las preferencias del grupo cuando las respuestas individuales son consistentes.

Dado que el método AHP discrimina más que la escala de Likert el peso que los alumnos atribuyen a los exámenes resueltos domina a todos los demás, tal y como se observa en la figura 3. Para analizar si las diferencias por grupos son significativas hemos realizado el test de Kruskal-Wallis. En caso afirmativo el test de Mann-Whitney nos permite detectar entre qué grupos existen diferencias significativas.

Cuando los alumnos comparan los cuatro tipos de recursos sólo es significativo el libro, debido al diferente comportamiento del grupo DAT con los grupos de mañana y tarde. En 
el análisis de las comparaciones sin incluir exámenes, resulta significativo el diferente comportamiento del grupo DAT con los otros tres grupos por el mayor uso de las presentaciones tal y como muestra la figura 4 .

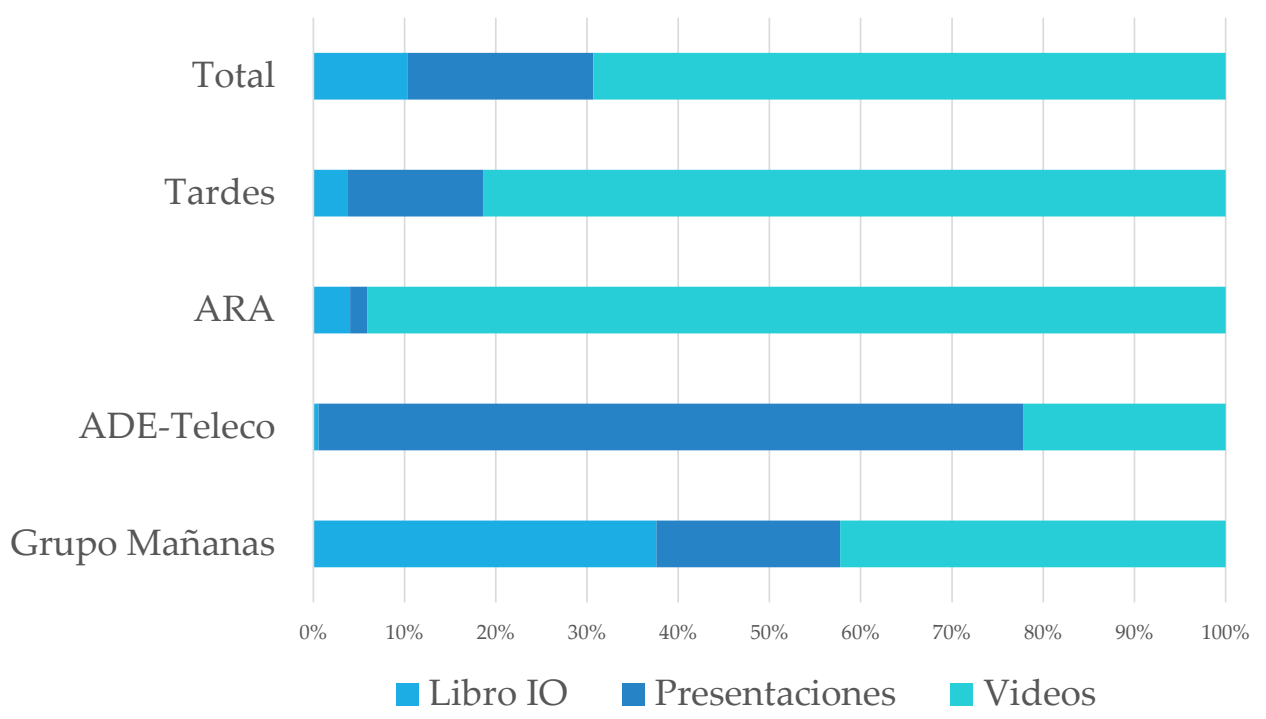

Fig. 4 Preferencias agregadas de los alumnos por el material docente en el aprendizaje de Investigación Operativa obtenidas con AHP. El porcentaje indica el peso relativo de cada uno de los 3 recursos.

También se han encontrado diferencias significativas entre las comparaciones por pares de matrices consistentes, cuando los alumnos comparan los videos con presentaciones y los videos con exámenes. En ambos casos el comportamiento del grupo de la doble titulación presenta un comportamiento distinto a los demás, dando menor preferencia a los videos que a las presentaciones cuando se comparan entre sí y también cuando se comparan con los exámenes.

Analizando la relación entre la calificación obtenida en la primera mitad de la asignatura y los materiales utilizados sólo son significativas las diferencias con los videos de unidades didácticas al 99 \% y con los videos de casos prácticos al 95\%. En el primer caso, el mayor porcentaje de alumnos que utilizan bastante o mucho los videos está en los que sacan notable, seguido de los aprobados. Asimismo, destaca que el 57\% de los sobresalientes no ven este tipo videos.

En cuanto a los videos de casos prácticos, un tercio de los suspensos los ha usado, mientras que la proporción sube al $56 \%$ en los aprobados y al $64 \%$ en los notables. Por otra parte, sólo los han visto el 28,6 \% de los sobresalientes. Atendiendo a este resultado se puede decir que los videos de casos prácticos son útiles para alcanzar un conocimiento mínimo y medio, aunque para alcanzar el mayor grado de aprendizaje y aprovechamiento se necesitan otras estrategias. 
También resulta significativo al 98\% la relación entre la meta personal en la calificación de la asignatura y la utilización de exámenes. Entre los que tienen una meta de notable un $85 \%$ utiliza mucho los exámenes, siendo este porcentaje menor en los demás casos.

El 72\% de las encuestas corresponden a alumnos que asisten regularmente a clase de teoría y prácticas de aula, ya que prácticamente todos asisten al laboratorio, que no siendo obligatorio, el trabajo que se realiza representa la evaluación continua del curso. El grado de utilización del libro presenta un comportamiento diferente y significativo dependiendo de si el alumno asiste o no a la clase presencial. Los que no van a clase usan el libro bastante o mucho $(41,1 \%)$ algo más que los que asisten $(35,6 \%)$. Sin embargo, la diferencia más importante está en que 1 de cada 3 de los que no van a clase no utilizan el libro, mientras este comportameinto sólo se da en el $17 \%$ de los que van a las clases presenciales.

La asistencia o no a clase presencial resulta significativa al 99\% de confianza con la visualización de videos con las explicaciones de las unidades didácticas. Un 74,3\% de los que no asisten a clase utilizan bastante o mucho estos videos y todos los ven, aunque sea muy poco. Por otra parte, el $45,8 \%$ de los que asisten a clase regularmente ven bastante o mucho estos videos y un $22 \%$ no los usa.

Por último, la asistencia regular a clase también está relacionada con la nota de la asignatura. Casi la mitad de los que no van a clase no han superado la primera parte de la asignatura (47,1\%), mientras que este dato es del $22 \%$ para los que sí asisten, que además de aprobar tienen mejores notas. En cuanto a los motivos por los que no asisten regularmente a clase, dos de cada tres contestan que prefieren ver los videos con las explicaciones de los temas, mientras que sólo la cuarta parte indica su preferencia por las presentaciones. El trabajo o realizar prácticas en empresas es el motivo de no asistir a clase en el 8,5\% de los casos.

Los resultados anteriores pueden estar relacionados con el comportamiento de muchos alumnos de dejar el trabajo para el final, como se ha podido comprobar con la evolución de las visualizaciones de los videos de casos prácticos. La figura 5 muestra que la mitad de las visitas se concentra en la semana anterior al examen, comportamiento general en todos los videos y documentado también por otros autores (Aljarrah et al. 2018).

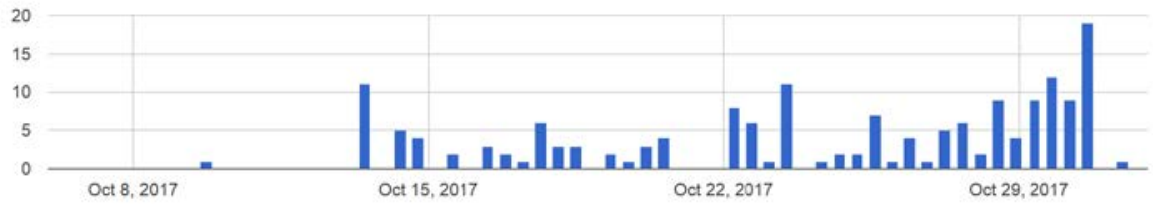

Fig. 5 Evolución del número de visitas (171) de un caso práctico (Algoritmo del simplex: método de las dos fases).

En cuanto a las sugerencias de mejora del material docente lo más destacable es que aproximadamente el $20 \%$ de los encuestados proponen disponer de más casos prácticos resueltos, incluyendo tanto los que especifican en formato video como los que no indican nada al respecto. El 2\% de los alumnos proponen incluir más casos reales en la asignatura. 


\section{Conclusiones}

Se ha constatado que todos los recursos disponibles para aprender Investigación Operativa que incluyen libros, presentaciones, videos y exámenes resueltos se utilizan por un número importante de alumnos. En general, los estudiantes muestran una clara preferencia por los exámenes resueltos, que utilizan y priorizan sobre videos, libros y presentaciones. Éste es un resultado que se ha obtenido con las dos metodologías, la escala de Likert y el método multicriterio AHP y además no está relacionado ni con el grupo ni con el rendimiento de los alumnos. Los exámenes resueltos con explicaciones detalladas mejoran el aprendizaje de los alumnos y reducen las tutorías.

El recurso preferido en segundo lugar son los videos, que utilizan bastante o mucho la mitad de los estudiantes y en una proporción mayor que el libro y las presentaciones del profesor, que no por esto dejan de ser relevantes. Las diferencias significativas por grupos se encuentran entre los alumnos de cursan la doble titulación con los demás grupos, tanto en los resultados de la escala de Likert como en los de AHP. Una mayor formación matemática, mayor regularidad en la asistencia y trabajo en clase y laboratorio, así como menor número de alumnos pueden estar en el origen de su menor empleo de videos para el aprendizaje.

Otra conclusión relevante es que la existencia de videos con las explicaciones de las unidades didácticas reducen la asistencia a clase, que está positivamente relacionada con el rendimiento académico. Por otra parte, reducen la necesidad de tutorías y favorecen el aprendizaje autónomo del alumno presencial. Sin embargo, los videos de casos prácticos interactivos presentan un uso más general por todos los alumnos y presentan una relación positiva con el rendimiento.

Por último, los resultados obtenidos no se pueden generalizar a cualquier titulación, pero consideramos que pueden ser representativos para los grados de ADE. La creciente demanda de contenidos online y de videos en particular sugieren que debemos seguir avanzando en el desarrollo de recursos especialmente diseñados para mejorar el aprendizaje y rendimiento de los alumnos, tales como sistemas de evaluación y videos interactivos de corta duración centrados en casos prácticos, vinculados entre sí ya que juegan un papel destacado en las preferencias y en el aprendizaje efectivo.

\section{Referencias}

ALCARAZ, J. et al. (2003). “Autonomous web-based learning of Operations Research” en International Conference on Engineering Education ICEE2003. Abstracts. Valencia.

ALCARAZ, J. et al. (2004). “Teaching Innovation of Operations Research Through a Web-Based System” en 7th Internacional Workshop on Higher Education and its Perspectives. Actas. Havana (Cuba).

ALJARRAH, A. et al. (2018). "Investigating temporal access in a flipped classroom: procrastination persists” en International Journal of Educational Technology in Higher Education 15:1, 1-18.

(cc) EY-NC-ND 2018, Universitat Politècnica de València

Congreso IN-RED (2018) 
ARBAUGH, J.B. et al. (2009). "Research in online and blended learning in the business disciplines: Key findings and possible future directions” en Internet and Higher Education 12, 71-87.

BAS, M.C. et al. (2017). "Preferencias en el aprendizaje de Investigación Operativa: Docencia inversa y presencial” en Congreso Nacional de Innovación Educativa y Docencia en Red (IN-RED 2017). Valencia, Spain: Editorial UPV. DOI:10.4995/INRED2017.2017.6806

COCHRAN, J.J. (2012). "You want them to remember? Then make it memorable! Means for enhancing operations research education” en European Journal of Operational Research 219, 659670.

GARUTI, C. (2017). "Reflections on scales from measurement, not measurements from scales” en International Journal of the Analytic Hierarchy Process 9, 349-361.

MAROTO, C. et al. (2012). Investigación Operativa en Administración y Dirección de Empresas. Valencia: Editorial UPV. Disponible en papel y libro electrónico.

MAROTO, C. et al. (2014). Operations Research in Business Administration and Management. Valencia: Editorial UPV. Disponible en papel y libro electrónico.

MAROTO, C. et al. (1997). "Operational Research Teaching in Computer Science through Six Years of Innovative Education Experience” en XV EURO and XXXIV INFORMS Joint International Meeting: OR/MS for the New Millennium. Abstracts.

SAATY, T.L. y PENIWATI, K. (2008). Group decision making: drawing out and reconciling differences. RWS Publications.

SAATY, T.L. y VARGAS, L.G. (2001). Models, methods, concepts \& applications of the analytic hierarchy process. Kluwer Academic Publishers.

SHARKEY, T.C. y NURRE, S.G. (2016). "Video Tutorials Within an Undergraduate Operations Research Course: Student Perception on Their Integration and Creating A Blended Learning Environment” en INFORMS Transactions on Education 17(1):1-12.

WINCH, J.K y CAHN, E.S. (2015). "Improving Student Performance in a Management Science Course With Supplemental Tutorial Videos” en Journal of Education for Business, 90:7, 402-409. 


\section{ANEXO. CUESTIONARIO}

\section{Proyecto de Innovación y Mejora Educativa}

PIME B01- curso 2017-18

\section{Preferencias sobre el material docente de Investigación Operativa}

El objetivo esta encuesta es conocer las preferencias de los alumnos por los diferentes tipos de materiales docentes disponibles para la asignatura Investigación Operativa.

\section{Indica el grupo en el que estás matriculado/a:}

\section{Grupo Mañanas}

Grupo Tardes

Grupo ARA

Grupo Doble ADE-Teleco

2. Grado de utilización del material docente en el aprendizaje de Investigación Operativa.

\begin{tabular}{|c|c|c|c|c|c|}
\hline Material docente & $\begin{array}{r}1 \\
\text { Nada }\end{array}$ & $\begin{array}{c}2 \\
\text { Muy } \\
\text { poco }\end{array}$ & $\begin{array}{c}3 \\
\text { Poco }\end{array}$ & $\begin{array}{c}\mathbf{4} \\
\text { Bastante }\end{array}$ & $\begin{array}{c}5 \\
\text { Mucho }\end{array}$ \\
\hline Biblioteca/Personal & & & & & \\
\hline Libro: Investigación Operativa en ADE & & & & & \\
\hline Book: Operations Research in BAM & & & & & \\
\hline En Poliforma T/Recursos: & & & & & \\
\hline Presentaciones de clase & & & & & \\
\hline Videos de casos prácticos & & & & & \\
\hline Exámenes resueltos & & & & & \\
\hline Videos Temas 2 y 3 (English) & & & & & \\
\hline PoliformaT/Contenidos: & & & & & \\
\hline $\begin{array}{l}\text { Videos con explicación de unidades } \\
\text { didácticas }\end{array}$ & & & & & \\
\hline
\end{tabular}


3. Indica tus preferencias por los distintos tipos de materiales docentes aplicando el método AHP basado en comparaciones por pares y la escala de 1 a 9 .

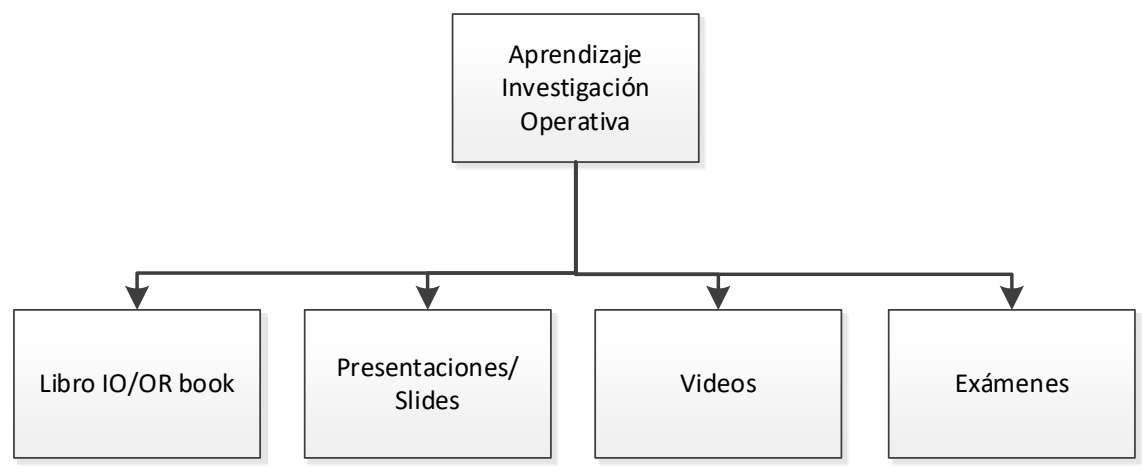

Compara la importancia relativa del material docente indicado en la fila de la matriz de comparaciones con el de la columna utilizando la escala del método AHP.

\section{Escala:}

1 igual importancia, 3 importancia moderada, 5 importancia fuerte, 7 mucha mayor importancia y 9 importancia extrema del primer elemento sobre el segundo.

Si el material de la columna tiene para ti mayor importancia que el de la fila: $1 / 3,1 / 5,1 / 7$ y $1 / 9$.

Rellena sólo las casillas en blanco.

\begin{tabular}{|c|c|c|c|c|}
\hline Material docente & Libro 10 & Presentaciones & Videos & Exámenes \\
\hline \multicolumn{5}{|l|}{ Libro 10} \\
\hline \multicolumn{5}{|l|}{ Presentaciones } \\
\hline \multicolumn{5}{|l|}{ Videos } \\
\hline Exámenes & & & & \\
\hline
\end{tabular}

Si tienes dudas sobre cómo rellenar esta matriz de comparaciones, contesta a las preguntas de la página siguiente. 


\begin{tabular}{|c|c|}
\hline Libro IO/OR book & Presentaciones/Slides \\
\hline $\begin{array}{l}\circ \mathbf{2} \\
\circ \text { Moderada = } 3 \\
\circ 4 \\
\circ \text { Fuerte = } 5 \\
\circ 6 \\
\circ \text { Mucha mayor = } 7 \\
\circ 8 \\
\circ \text { Extrema = } 9\end{array}$ & $\begin{array}{l}\circ \mathbf{2} \\
\circ \text { Moderada }=3 \\
\circ 4 \\
\circ \text { Fuerte }=5 \\
\circ 6 \\
\circ \text { Mucha mayor }=7 \\
\circ 8 \\
\circ \text { Extrema }=9\end{array}$ \\
\hline
\end{tabular}

¿Cuál de los dos materiales docentes tiene para ti más importancia en el aprendizaje de Investigación Operativa?

\begin{tabular}{|c|c|}
\hline Libro IO/OR book & Exámenes \\
\hline $\begin{array}{ll} & \mathbf{2} \\
\circ & \text { Moderada = } 3 \\
\circ & 4 \\
\text { - } & \text { fuerte }=5 \\
6 & 6 \\
\text { - Mucha mayor = } 7 \\
-8 \\
\text { - Extrema }=9\end{array}$ & $\begin{array}{l}\circ \mathbf{2} \\
\circ \text { Moderada = } 3 \\
\circ \mathbf{4} \\
\circ \text { Fuerte = } 5 \\
\circ 6 \\
\circ \text { Mucha mayor = } 7 \\
\circ 8 \\
\circ \text { Extrema = } 9\end{array}$ \\
\hline
\end{tabular}

¿Cuál de los dos materiales docentes tiene para ti más importancia en el aprendizaje de Investigación Operativa?

\begin{tabular}{|c|c|}
\hline Presentaciones & Exámenes \\
\hline $\begin{array}{ll}\circ & \mathbf{2} \\
\circ & \text { Moderada }=\mathbf{3} \\
\circ & 4 \\
\circ & \text { Fuerte }=\mathbf{5} \\
\circ & 6 \\
\circ & \text { Mucha may = } 1 \\
\circ & 8 \\
\circ & \text { Extrema }=\mathbf{9}\end{array}$ & $\begin{array}{ll}\circ & \mathbf{2} \\
\circ & \text { Moderada = } 3 \\
\circ & 4 \\
\circ & \text { Fuerte }=\mathbf{5} \\
\circ & 6 \\
\circ & \text { Mucha mayor = } 7 \\
\circ & 8 \\
\circ & \text { Extrema }=9\end{array}$ \\
\hline
\end{tabular}

¿Cuál de los dos materiales docentes tiene para ti más importancia en el aprendizaje de Investigación Operativa?

\begin{tabular}{|c|c|c|}
\hline Libro IO/OR book & & Videos \\
\hline$\circ 2$ & ○ Igual = 1 & $\circ 2$ \\
\hline - Moderada $=\mathbf{3}$ & & - Moderada $=\mathbf{3}$ \\
\hline$\circ 4$ & & $\circ 4$ \\
\hline ○ Fuerte $=\mathbf{5}$ & & ○ Fuerte $=\mathbf{5}$ \\
\hline$\circ 6$ & & $\circ 6$ \\
\hline - Mucha mayor $=7$ & & - Mucha mayor $=7$ \\
\hline$\circ 8$ & & $\circ 8$ \\
\hline$\circ$ Extrema $=\mathbf{9}$ & & ○ Extrema $=\mathbf{9}$ \\
\hline
\end{tabular}

¿Cuál de los dos materiales docentes tiene para ti más importancia en el aprendizaje de Investigación Operativa?

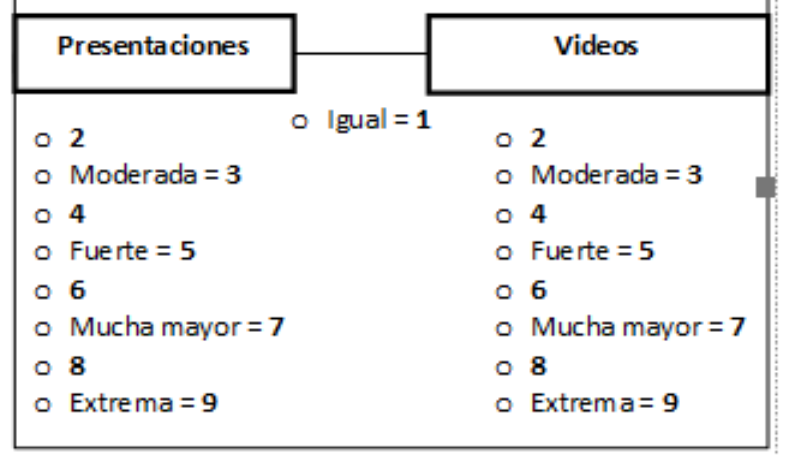

¿Cuál de los dos materiales docentes tiene para ti más importancia en el aprendizaje de Investigación Operativa?

\begin{tabular}{|c|c|c|c|c|}
\hline & Videos & & & Exámenes \\
\hline$\circ$ & 2 & - Igual $=1$ & 0 & 2 \\
\hline$\circ$ & Moderada $=\mathbf{3}$ & & 0 & Moderada $=\mathbf{3}$ \\
\hline$\circ$ & 4 & & $\circ$ & 4 \\
\hline$\circ$ & Fuerte $=\mathbf{5}$ & & 0 & Fuerte $=\mathbf{5}$ \\
\hline$\circ$ & 6 & & 0 & 6 \\
\hline$\circ$ & Mucha mayor $=\mathbf{7}$ & & 0 & Mucha mayor $=7$ \\
\hline$\circ$ & 8 & & $\circ$ & 8 \\
\hline 0 & Extrema $=\mathbf{9}$ & & 0 & Extrema $=\mathbf{9}$ \\
\hline
\end{tabular}


4. Calificación en la primera parte de la asignatura (Examen + Prácticas laboratorio):

No presentado

Suspenso

Aprobado

Notable

Sobresaliente

5. Asisto regularmente a las clases:

Teóricas y Prácticas de aula

SI

NO

\section{Prácticas Informáticas}

SI

NO

En caso de NO asistencia indica el motivo:

Prácticas de empresa

Prefiero ver los videos con explicación de los temas

Prefiero preparar la asignatura con el libro

Prefiero estudiar con las presentaciones de clase

Otros:

6. Meta personal para la calificación final de la asignatura Investigación Operativa

Aprobado

Notable

Sobresaliente

Sobresaliente $10 \mathrm{MH}$

7. Sugerencias de mejora para la elaboración de materiales docentes:

(c) BY-NC-ND 2018, Universitat Politècnica de València

Congreso In-Red (2018) 